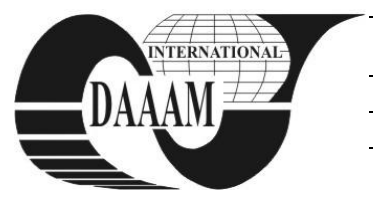

Annals of DAAAM for 2011 \& Proceedings of the 22nd International DAAAM Symposium, Volume 22, No. 1, ISSN 1726-9679 ISBN 978-3-901509-83-4, Editor B. Katalinic, Published by DAAAM International, Vienna, Austria, EU, 2011 Make Harmony between Technology and Nature, and Your Mind will Fly Free as a Bird Annals \& Proceedings of DAAAM International 2011

\title{
APPLICABILITY OF ANALYTICAL TREE SEARCH ALGORITHMS IN NETWORK ROUTING
}

\author{
CAVAR, I[van] \& VALENCIC, D[avorin]
}

\begin{abstract}
Today's network routing protocols use various algorithms for finding the best path to other networks. For example, we have routing protocols that use Bellman-Ford, Dijsktra's Shortest Path First and Cisco's Diffusing Update Algorithm. In this article we will analyze if there are any other tree search algorithms that can be used in network routing and what to expect from them.
\end{abstract}

Key words: routing, routing protocols, SPF, A*, FloydWarshall

\section{INTRODUCTION}

Network routing is an essence of today's IP (Internet Protocol) networks, where routers are forwarding IP packets among networks. Router needs to know network topology or at least one part of that topology, to be able to know where the packet should be forwarded. Based on the information they acquire from knowing the network topology, routers can build their forwarding tables which they use for forwarding packets.

Today's most commonly used protocol in Autonomous Systems is OSPF (Open Shortest Path First) (RFC 2328) protocol which is based on Dijkstra's SPF (Shortest Path First) algorithm. Dijkstra's SPF algorithm (Dijkstra, 1959) is "Single source - All destination" algorithm known as accurate, but greedy algorithm. As a result of that we get high CPU and memory intensive algorithm for routers in large network topologies. Each time a network topology changes, SPF algorithm need to be recomputed and due to the errors and link flapping in networks, that could happen very often. Frequent SPF recomputation leads to delays in forwarding and packet dropping on routers. OSPF is not the only routing protocol that uses SPF algorithm, OSI developed IS-IS (Intermediate System - Intermediate System) (RFC 1195) protocol that also uses Dijkstra's SPF algorithm.

Another routing protocol that we use for smaller networks is RIP (Routing Information Protocol) (RFC 2453), that uses Bellman-Ford algorithm. A problem with that algorithm is that it is unweighted so we get imprecise information about network distance. The only metric we have is the number of hops, or the number of routers to the destination network. Besides these routing protocols, Cisco Systems developed a proprietary one named EIGRP (Enhanced Interior Gateway Routing Protocol) which is based on DUAL-FSM (Diffusing Update Algorithm Finite State Machine). Since EIGRP is a proprietary routing protocol, we will not consider it further more in this paper. The same goes for DUAL-FSM algorithm.

Except these tree search algorithms, we have many other algorithms that we can use with routing protocols. We will analyze if we can use some of them for today's routing protocols or maybe for some future ones. In these article we will focus on modified version of SPF algorithm that uses Fibonacci heaps, A* (star) algorithm and Floyd Warshall algorithm. Other algorithms are not examined because most of them are not designed to solve problems like the ones we have in network routing.

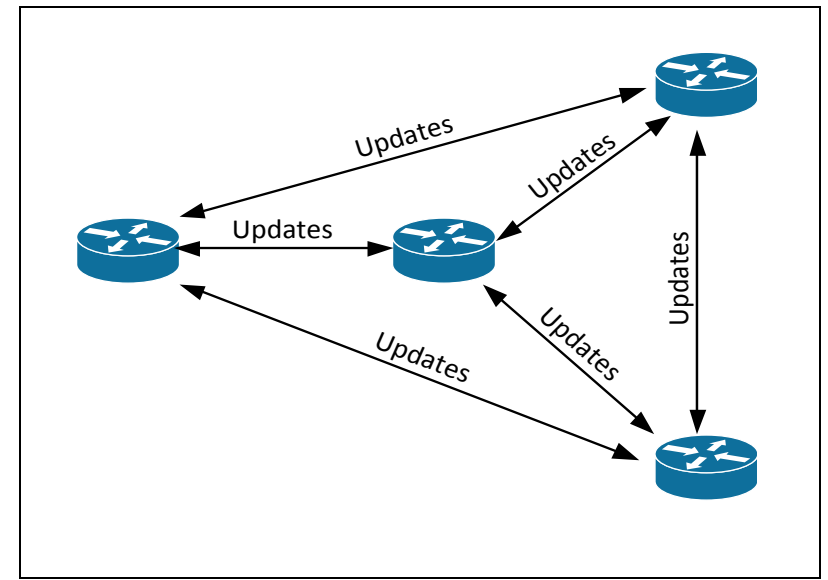

Fig. 1. Updates from router traversing to every other router

On the other hand, network routing with current routing protocols is conceptually solved in a way that network information from one router is traversing to every other router in a network (except across summarization point) as we can see in Figure 1.We will briefly analyze and propose implementation of a new model in router communication process with usage of other algorithms.

\section{APLICABLE ALGORITHMS FOR NETWORK ROUTING}

\subsection{Dijkstra's algorithm with Fibonacci heaps}

Dijsktra algorithm has been improved in 1984 by implementing Fibonacci heaps in SPF algorithm (Fredman \& Tarjan, 1987). As a result we gain an implementation of SPF with priority queue and much faster computations. Use of SPF with Fibonacci heaps doesn't require any change in existing routing protocols that use SPF, like OSPF and IS-IS, because computations are local on each router.

\section{$2.2 \mathrm{~A} *$ (A star) algorithm}

A* (A star) algorithm (Dechter \& Pearl, 1985) is based on Dijkstra SPF algorithm and it is known as the fast and accurate algorithm, which achieves its performance by using heuristics. With $\mathrm{A}^{*}$ algorithm all routers in one topology area should share all of the network information, like in OSPF or IS-IS that use SPF algorithm. If we use $A^{*}$ for network routing we get the following result:

- Faster route calculation

- Less CPU and memory overhead for routers

- Since algorithm searches for the least cost to the destination using heuristics, there could be a problem with load balancing through multiple equal cost paths

- Possible implementation of algorithm with no need for complete topology recomputation when a change in topology occurs. 
A* star is suitable for implementation in existing routing protocols like OSPF, but protocol itself should have some changes to adopt the new possibilities of $A^{*}$ algorithm.

There are many variants of $A^{*}$ algorithm that could offer new possibilities like D* (Dynamic A*) (Stentz, 1995) where cost can be changed when algorithm runs. With that functionality we can implement dynamic updates with no need for recomputation of complete algorithm like we have with original Dijsktra SPF algorithm.

\subsection{Floyd-Warshall algorithm}

Floyd-Warshall algorithm (Cormen et al., 1990) is "all pairs" algorithm that searches all paths between all nodes. If we want to use this algorithm in network routing we have two approaches. In one approach all routers use this algorithm, which is not a good solution because routers only need to know their own distance to remote networks.

Other approach is that we use one server router that will do all computation in the network for every client router, and then send the results to the client routers in the form of routing tables. In that way, we have client-server relationship between routers, where server became "brain" router for that specific area or topology (Figure 2).

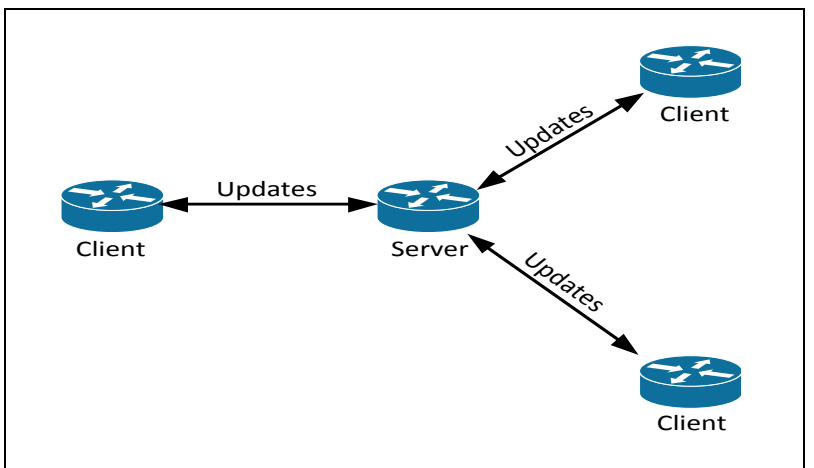

Fig. 2.Client-Server model in routing updates

If we try to use client-server model in routing protocol, we get similar situation like the one we have on broadcast multiaccess segment in OSPF with DR (Designated Router) model, where one DR updates all the other non-DR routers, and nonDR routers update only DR routers. Problem will arise if a server becomes unreachable, so there is a need for constant backup mechanism for the server router. Client routers also need a constant connection with the server router on the network layer, so they can exchange updates directly.

\section{GUIDELINES FOR FURTHERROUTING PROTOCOL DEVELOPMENT}

In today's IP networks we have IGP-s (Interior Gateway Protocols) that work in Autonomous System (like RIP, OSPF, IS-IS, EIGRP) and we have only one Exterior Gateway Protocol, BGP (Border Gateway Protocol) that connects all Autonomous Systems and bind them into global network Internet.

Since there are already a number of implemented IGP protocols and a lot of issues related to their incompatibility to work together in the same AS, there is no much sense in adding one more interior gateway protocol. Instead we have option for upgrading existing routing protocol with faster algorithms like A* in OSPF protocol.

For easy upgrade of existing protocols, vendors of network equipment could implement SPF with Fibonacci heaps and gain better performance for their routers and keep full interoperability with other routers from other vendors.
When implementing $\mathrm{A}^{*}$ algorithm, there will need to be written another Request for Comment on existing routing protocols on Internet Engineering Task Force, and proposal should be accepted before vendors implement it on their equipment. A better solution would be to build a whole new routing protocol that will set out all the problems with existing protocols and possibly integrate whole routing in one global, hierarchical, and level based routing protocol.

Floyd-Warshall algorithm is interesting for the new concept of IP routing, where client routers don't need a lot of intelligence for finding the best path to certain network. Instead, we can create a whole new concept of centralized routing solution where one server router calculates a best path for all the networks and pass them to every client router in the network. This kind of solution could save a lot of resource needed on routers and make them more efficient in routing process. They will not be overloaded with route computation and update sharing with all the neighboring routers. Also from the administration point of view, there is no need for complex configuration of all routers in the network, basically we only need to configure router that will be server router.

\section{CONCLUSION}

Today's network routing is a very complex area with a lot of different routing protocols inside every Autonomous system, where every router directly communicates with all it's neighbors to exchange updates. These routing protocols are based on elemental Dijsktra's SPF algorithm and Bellman-Ford algorithm. We analyzed the usage of other tree search algorithms that could help us in solving some routing issues with possible upgrade or new routing protocol. We found out that improved version of Dijsktra's SPF with Fibonacci heaps can be easily adopted without any major change of existing routing protocols. On the other hand $A^{*}$ is an excellent algorithm for new routing protocol and Floyd-Warshall algorithm can be used for new routing concept in server-client mode. These conclusions can be a good base for further research in a network routing concepts.

\section{REFERENCES}

Cormen, Thomas H.; Leiserson, Charles E., Rivest, Ronald L. (1990). Introduction to Algorithms (1st ed.). MIT Press and McGraw-Hill. ISBN 0-262-03141-8

Dechter, Rina; Pearl, Judea (1985).Generalized best-first search strategies and the optimality of $A *$. Journal of the ACM 32 (3): 505-536. doi:10.1145/3828.3830

Dijkstra, E. W. (1959). A note on two problems in connexion with graphs. Numerische Mathematik 1: 269-271. doi:10.1007/BF01386390

Fredman, Michael Lawrence; Tarjan, Robert E. (1987).Fibonacci heaps and their uses in improved network optimization algorithms. Journal of the Association for Computing Machinery 34 (3): 596-615. doi:10.1145/28869.28874

Internet Engineering Task force (April 1998); OSPF Version 2; RFC 2328 Available from: http://www.ietf.org/rfc/rfc2328.txt Accessed: 2011-06-25

Internet Engineering Task force (November 1998); RIP Version 2; RFC 2453 Available from: http://www.ietf.org/rfc/rfc2453.txt Accessed: 2011-06-25

Internet Engineering Task force (December 1990); Use of OSI IS-IS for Routing in TCP/IP and Dual Environments; RFC 1195 Available from: http://www.ietf.org/rfc/rfc1195.txt Accessed: 2011-06-25

Stentz, Anthony (1995), TheFocussed D* Algorithm for RealTime Replanning, In Proceedings of the International Joint Conference on Artificial Intelligence: 1652-1659 